\title{
Fibroscan improves the diagnosis sensitivity of liver fibrosis in patients with chronic hepatitis B
}

\author{
RENGANG HUANG ${ }^{1}$, NAN JIANG ${ }^{1}$, RENGUO YANG ${ }^{1}$, XIAOXIA GENG ${ }^{1}$, JIANMEI LIN ${ }^{1}$, GANG XU $^{2}$, \\ DANDAN LIU ${ }^{2}$, JIDOG CHEN $^{3}$, GUO ZHOU ${ }^{3}$, SHUQIANG WANG ${ }^{1}$, TINGTING LUO ${ }^{1}$, \\ JIAZHEN WU ${ }^{1}$, XIAOSHU LIU ${ }^{1}$, KAIJU XU ${ }^{1}$ and XINGXIANG YANG ${ }^{1}$ \\ Departments of ${ }^{1}$ Infectious Disease, ${ }^{2}$ Pathology and ${ }^{3}$ Medical Ultrasonics, \\ Sichuan Academy of Medical Sciences and Sichuan Provincial People's Hospital, Chengdu, Sichuan 610072, P.R. China
}

Received October 28, 2014; Accepted October 22, 2015

DOI: $10.3892 / e t m .2016 .3135$

\begin{abstract}
The aim of the present study was to investigate the diagnostic accuracy of Fibroscan for liver fibrosis in patients with chronic hepatitis $\mathrm{B}(\mathrm{CHB})$ with alanine aminotransferase (ALT) levels $<2$ times the upper normal limit. A total of 263 consecutive patients with CHB and ALT levels $<2$ times the upper normal limit were enrolled in the present study. Liver biopsies and liver stiffness measurements (LSM) were conducted. Receiver operating characteristic (ROC) analysis was used to determine the predictive ability of LSM for the development of liver fibrosis in patients with stage S1, S2 and S3 liver fibrosis. Bivariate Spearman rank correlation analysis was performed in order to determine the association between liver stiffness value, which was measured by Fibroscan, and liver fibrosis stage, which was measured by liver biopsy. The liver stiffness value was found to be positively correlated with the liver fibrosis stage $(\mathrm{r}=0.522, \mathrm{P}<0.001)$ and necroinflammatory activity $(\mathrm{r}=0.461, \mathrm{P}<0.001)$, which was measured by liver biopsy. The optimal cut-off value in the patients with stage $\mathrm{S} 1$, $\mathrm{S} 2$ and S3 liver fibrosis was 5.5, 8.0 and $10.95 \mathrm{kPa}$, respectively. The area under the ROC curve for the prediction of the development of liver fibrosis in these patients was 0.696, 0.911 and 0.923 , respectively. The threshold of the optimal cut-off value exhibited a high sensitivity and specificity. The results of the present study suggested that Fibroscan may improve the sensitivity of the diagnosis of liver fibrosis in patients with CHB and ALT levels $<2$ times the upper normal limit, and that this sensitivity may increase with the progression of liver fibrosis.
\end{abstract}

Correspondence to: Dr Xingxiang Yang, Department of Infectious Disease, Sichuan Academy of Medical Sciences and Sichuan Provincial People's Hospital, 32 West Second Section First Ring Road, Chengdu, Sichuan 610072, P.R. China

E-mail: ygan333@163.com

Key words: fibroscan, chronic hepatitis B, liver fibrosis, liver stiffness measurement

\section{Introduction}

Chronic liver disease comprises a process of progressive destruction and regeneration of the liver parenchyma (1). Liver fibrosis is known to result from chronic liver damage in conjunction with the excessive accumulation of extracellular matrix proteins, a characteristic of the majority of chronic liver disease types (2). Chronic infection with hepatitis B virus (HBV) remains the predominant cause of chronic liver disease and liver-related morbidity worldwide (3). Asia and the Western Pacific have the highest proportion of global chronic hepatitis $\mathrm{B}(\mathrm{CHB})$ cases, with $75 \%$ of all CHB patients concentrated in these countries (4). CHB is considered to be the major risk factor for cirrhosis, end-stage liver disease and hepatocellular carcinoma $(2,5)$. In the hepatic tissues of patients with $\mathrm{CHB}$, the accumulation of extracellular matrix proteins distorts the hepatic architecture by forming a fibrous scar (6). The subsequent development of nodules of regenerating hepatocytes eventually leads to liver cirrhosis (5). Therefore, it is crucial to achieve an accurate and timely diagnosis of liver fibrosis in order to prevent its development to liver cirrhosis.

Liver biopsy is a diagnostic procedure, which involves the examination of a small liver tissue sample for liver disease, and is considered to be the gold standard for the diagnosis of liver fibrosis (7). However, due to its invasiveness and high-cost, the application of liver biopsy in the evaluation of liver fibrosis is limited. The liver tissue samples obtained for liver biopsy comprise only $\sim 1 / 50,000$ of the entire liver tissue (8), and therefore may not reflect the condition of the entire liver. In previous studies, liver biopsies conducted using tissue samples $15-25 \mathrm{~mm}$ in length were consistent with histopathological examination results in $65-75 \%$ of cases $(9,10)$.

Liver stiffness measurement (LSM) using Fibroscan is a non-invasive, rapid, quantitative and low-cost transient elastographic method of assessing the degree of liver fibrosis (11). Briefly, vibrations of mild amplitude and low frequency are transmitted by the transducer, and induce an elastic shear wave that is propagated within the liver. Pulseecho ultrasonic acquisitions are performed in order to follow the shear wave and measure its speed, which is directly associated with the tissue stiffness (the harder the tissue, the faster the shear propagates). Results are expressed in Kilopascals (Kpa) and 
correspond to the median value of ten validated measurements ranging from 2.5 to $75 \mathrm{Kpa}$ (12). However, the results of LSM may be influenced by factors other than fibrosis, including necroinflammatory activity, obesity, extrahepatic cholestasis and sampling location (13-16). The efficacy of transient elastography has been validated in patients with chronic hepatitis C; however, limited data are available for its use in patients with other forms of liver disease, particularly patients with $\mathrm{CHB}$ and alanine aminotransferase (ALT) $<2$ times the upper normal limit. A previous study has suggested that the diagnostic accuracy of liver fibrosis as measured by Fibroscan may be influenced by the elevated ALT concentration (17).

Measurement of serum ALT levels is widely used for the assessment of liver diseases (18). The upper limit of normal ALT is considered to be a reliable standard to assess liver function (19). In the present study, the diagnostic accuracy of Fibroscan for liver fibrosis development in patients with CHB with ALT levels $<2$ times the upper normal limit was investigated.

\section{Materials and methods}

Patients. A total of 263 patients (160 males and 103 females; age range, 16-67 years; average age, $33.48 \pm 11.36$ years) with $\mathrm{CHB}$ from the Department of Infectious Diseases at the Sichuan Academy of Medical Sciences \& Sichuan Provincial People's Hospital (Chengdu, China) were enrolled in the present study between October 2010 and August 2013. The inclusion criteria were as follows: i) Tested positively for the Hepatitis B surface antigen (HBsAg); ii) ATL levels were sustained $<2$ times the normal upper limit for 6 months and did not receive drugs for the reduction of ALT levels (0-80 U/L); iii) received no antiviral therapy; and iv) were willing to undergo liver biopsy. The exclusion criteria were as follows: i) Infected with hepatitis A, $\mathrm{C}$ or $\mathrm{E}$ virus; ii) infected with the human immunodeficiency virus; iii) suffered from a type of chronic liver disease other than CHB; iv) total serum bilirubin level $>34.2 \mu \mathrm{M}$ (normal range, 1.71-17.10 $\mu \mathrm{M}$ ); and v) suffered from liver cirrhosis, as indicated by diagnostic imaging. Prior written and informed consent was obtained from each patient. The study was approved by the Ethics Review Board of the Sichuan Academy of Medical Sciences \& Sichuan Provincial People's Hospital.

LSM assay. LSM assays were conducted according to the instructions provided by the manufacturer of FibroScan 502 Touch (Echosens; Paris, France) using an M probe attachment. Briefly, measurements were performed following an overnight period of fasting. Mild amplitude and low-frequency vibrations $(50 \mathrm{~Hz})$ were transmitted to the liver of each patient, inducing an elastic shear wave propagating through the underlying liver tissue. The velocity of the wave directly correlated to the tissue stiffness. The tip of the transducer was covered with a drop of gel and placed perpendicularly in the intercostal space of the patient, who was required to lie in the dorsal decubitus position with the right arm on maximal abduction. Scanning was conducted in a region encompassing the 6th, 7th and 8th intercostal spaces between anterior axillary and midaxillary lines. The number of successfully detections per case was required to be $\geq 10$, as LSM may be considered reliable when 10 valid measurements are obtained, with a success rate of $\geq 80 \%$ and an interquartile range/median LSM $\leq 30 \%$ (20-22). The median value, expressed in $\mathrm{kPa}$, was recorded as representative of the liver stiffness.

Liver biopsy. Liver biopsies were performed percutaneously following 2-3 ml local anesthetic with $1 \%$ lidocaine, and samples were obtained using a biopsy instrument with a 16 G needle (BARD ${ }^{\circledR}$ MAGNUM $^{\circledR}$, Bard Biopsy Systems, Tempe, AZ, USA). The samples were fixed in formalin and embedded in paraffin. The embedded tissues were cut into 4- $\mu \mathrm{m}$ sections and stained with hematoxylin and eosin and Masson's trichrome stains (both Beisen, Inc., Nanjing, China). Liver fibrosis was semi-quantitatively evaluated according to the Batts-Ludwig scoring system (7). The activity grade referred to the grades of hepatic necroinflammatory activity as follows: G0, no activity; G1, minimal activity; G2, mild activity; G3, moderate activity; and G4, severe activity. Liver fibrosis in liver tissues was graded as follows: S0, no fibrosis, normal connective tissue; S1, portal fibrosis, fibrous portal expansion); S2, periportal fibrosis, periportal or rare portal-portal septa; S3, septal fibrosis, fibrous septa with architectural distortion, no obvious cirrhosis; and S4, cirrhosis (7).

HBsAg and DNA assay. HBsAg was detected using the HBsAg enzyme-linked immunosorbent assay (ELISA) kit (Wanti BioPharm, Inc., Beijing, China), according to the manufacturer's protocol. Serum HBV DNA levels were measured using Diagnostic kit for Quantification of $\mathrm{HBV}$ DNA (PCR-Fluorescence) (DaAn Gene Co.,Ltd., Guangzhou, China) with a lower limit detection of 1,000 copies $/ \mathrm{ml}$, according to the manufacturer's instructions.

ALT detection assay. Peripheral blood was collected from patients with $\mathrm{CHB}$ with ATL levels $<2$ times the normal upper limit. ALT was detected using the enzymatic method as described by Reitman and Frankel (23), using ALT detection reagents obtained from Zhongsheng Beikong Reagent, Co., Ltd. (Beijing, China). ALT levels were measured using the Automatic Biochemistry analyzer (AU2700; Olympus Corporation, Tokyo, Japan).

Statistical analysis. All results are expressed as the mean \pm standard deviation. All statistical analyses were performed using the SPSS, version 18.0 software (SPSS, Inc., Chicago, IL, USA). Receiver operating characteristic (ROC) analysis was used to determine the ability of LSM to predict the development of liver fibrosis in patients with CHB. The area under the curve was a calculated as an overall assessment of the predictive ability of LSM. Bivariate Spearman rank correlation coefficient was used to analyze the correlation between the LSM and histological fibrosis grade. $\mathrm{P}<0.05$ was considered to indicate statistically significant difference.

\section{Results}

General data of patients with $\mathrm{CHB}$. According to the inclusion and exclusion criteria, a total of 263 patients (160 males and 103 females) with CHB were enrolled in the present study. 
Table I. Liver enzyme levels in patients with chronic hepatitis B.

\begin{tabular}{lcccc} 
Liver enzyme & Range (U/l) & Levels (U/l) & $\begin{array}{c}\text { Cases with levels } \\
\leq 40 \mathrm{U} / 1(\mathrm{n})\end{array}$ & $\begin{array}{c}\text { Cases with levels } \\
41-80 \mathrm{U} / 1(\mathrm{n})\end{array}$ \\
\hline Alanine aminotransferase & $12-79$ & $44.07 \pm 16.84$ & 120 & 143 \\
Aspartate aminotransferase & $7-77$ & $38.57 \pm 10.81$ & 161 & 102 \\
\hline
\end{tabular}

Table II. Grade of necroinflammatory activity and liver fibrosis stage in patients with chronic hepatitis B.

\begin{tabular}{|c|c|c|c|}
\hline \multicolumn{2}{|c|}{ Necroinflammatory activity } & \multicolumn{2}{|c|}{ Liver fibrosis } \\
\hline Grade & Cases (\%) & Stage & Cases $(\%)$ \\
\hline G0 & $0(0)$ & So & $17(6.5)$ \\
\hline G1 & $79(30.0)$ & S1 & $207(78.7)$ \\
\hline G2 & $125(47.5)$ & S2 & $25(9.5)$ \\
\hline G3 & $52(19.8)$ & S3 & $11(4.2)$ \\
\hline G4 & $7(2.7)$ & S4 & $3(1.1)$ \\
\hline Total & $263(100)$ & Total & $263(100)$ \\
\hline
\end{tabular}

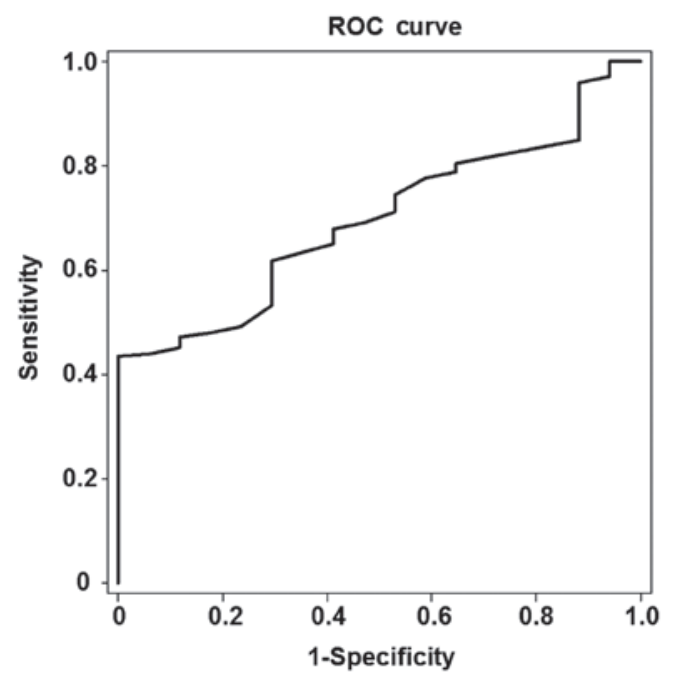

Figure 1. ROC curve for the analysis of liver stiffness measurement values, which were measured by Fibroscan, in the patients with stage S1 liver fibrosis. The area under the ROC curve for the prediction of the development of liver fibrosis was 0.696 , and the optimal cut-off value was $5.55 \mathrm{kPa}$ in the patients with stage S1 liver fibrosis. The threshold of the optimal cut-off value had a sensitivity of $61.8 \%$ and a specificity of $70.6 \%$. ROC, receiver operating characteristic.

The age range of the patients was 16-67 years, with an average age of $33.48 \pm 11.36$ years. The general data of liver enzyme levels of these patients are presented in Table I. The ALT level range of the patients was 12-79 $\mathrm{U} / \mathrm{l}$, with an average level of 44.07 $\pm 16.84 \mathrm{U} / 1$. Among the 263 patients, 120 had ALT levels of $\leq 40 \mathrm{U} / 1$ and $143 \mathrm{had}$ an ALT level range of 41-80 U/1. The aspartate aminotransferase (AST) level range was 7-77 U/1, with an average level of $38.57 \pm 10.81 \mathrm{U} / 1$. Among the 263 patients, 161 had AST levels of $\leq 40 \mathrm{U} / 1$ and 102 had an AST level range of 41-80 U/1. As shown in Table II, with regard to the grade of necroinflammatory activity, 125 cases $(47.5 \%)$ had mild portal

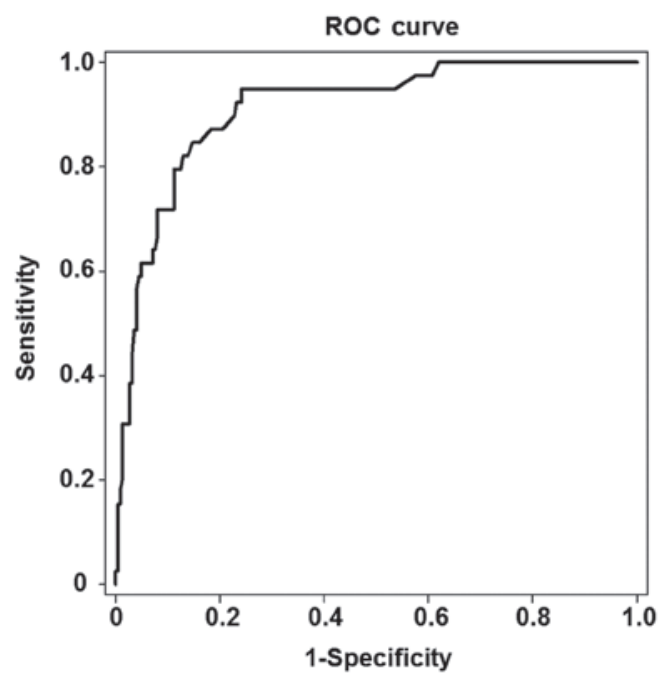

Figure 2. ROC curve for the analysis of liver stiffness measurement values, which were measured by Fibroscan, in the patients with stage S2 liver fibrosis. The area under the ROC curve for the prediction of the development of liver fibrosis was 0.911 , and the optimal cut-off value was $8.0 \mathrm{kPa}$ in the patients with stage S2 liver fibrosis. The threshold of the optimal cut-off value had a sensitivity of $86.4 \%$ and a specificity of $85.3 \%$. ROC, receiver operating characteristic.

inflammatory infiltrates (G2). According to the stage of liver fibrosis, 207 cases (78.7\%) had mild liver fibrosis (S1).

Correlation analysis between LSM values and liver fibrosis stage. In order to investigate the association between LSM values and the stage of liver fibrosis for the diagnosis of liver fibrosis in patients with CHB and ATL levels $<2$ times the normal upper limit, Bivariate Spearman rank correlation analysis was performed. The LSM values, which were measured by Fibroscan, positively correlated with the stage of liver fibrosis $(\mathrm{r}=0.522, \mathrm{P}<0.001)$, as well as the grade of 


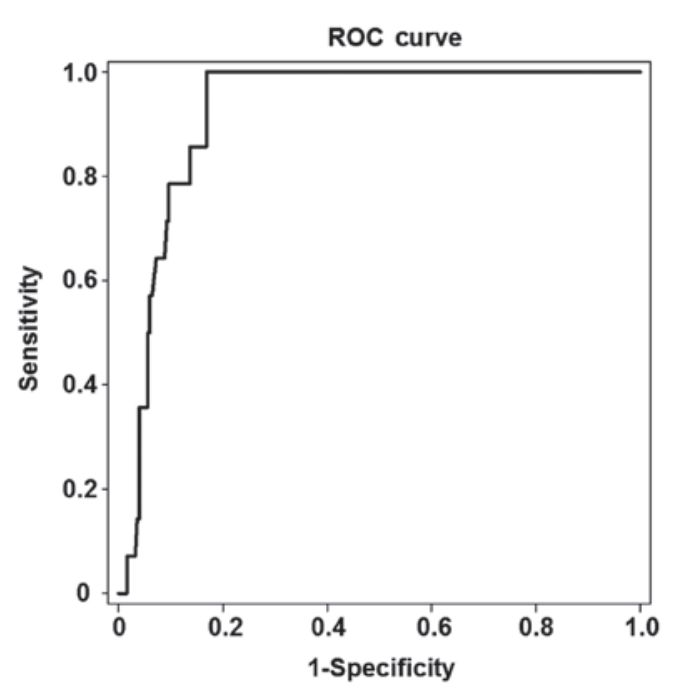

Figure 3. ROC curve for the analysis of liver stiffness measurement values, which were measured by Fibroscan, in the patients with stage S3 liver fibrosis. The area under ROC curve for the prediction of the development of liver fibrosis was 0.923 , and the optimal cut-off value was $10.95 \mathrm{kPa}$ in the patients with stage S3 liver fibrosis. The threshold of the optimal cut-off value had a sensitivity of $78.6 \%$ and a specificity of $90.4 \%$. ROC, receiver operating characteristic.

necroinflammatory activity $(\mathrm{r}=0.461, \mathrm{P}<0.001)$. The stage of liver fibrosis positively correlated with the grade of necroinflammatory activity $(\mathrm{r}=0.705, \mathrm{P}<0.001)$. These results indicate that the LSM values positively correlated with the degree of liver fibrosis and necroinflammatory activity.

ROC analysis for the prediction of liver fibrosis development in patients with $C H B$. In order to determine the diagnostic accuracy of Fibroscan for the prediction of liver fibrosis development in patients with $\mathrm{CHB}, \mathrm{ROC}$ analysis was performed. Among the 263 patients, 246 were found to have stage S1-S4 liver fibrosis and 17 had stage S0 liver fibrosis. As shown in Fig. 1, the area under the ROC curve for the prediction of liver fibrosis development was 0.696, and the optimal cut-off value was $5.55 \mathrm{kPa}$ in the patients with stage $\mathrm{S} 1$ liver fibrosis $(\mathrm{P}<0.05)$. The threshold of the optimal cut-off value had a sensitivity of $61.8 \%$ and a specificity of $70.6 \%$.

Among the 263 patients, 39 were found to have stage S2-S4 liver fibrosis and 224 stage S0-S1 liver fibrosis. As shown in Fig. 2, the area under the ROC curve for the prediction of liver fibrosis development was 0.911 , and the optimal cut-off value was $8.0 \mathrm{kPa}$ in the patients with stage $\mathrm{S} 2$ liver fibrosis $(\mathrm{P}<0.05)$. The threshold of the optimal cut-off value had a sensitivity of $86.4 \%$ and a specificity of $85.3 \%$.

Among the 263 patients, 14 were found to have stage S3-S4 and 249 stage S0-S2 liver fibrosis. As shown in Fig. 3, the area under the ROC curve for the prediction of liver fibrosis development was 0.923 , and the optimal cut-off value was $10.95 \mathrm{kPa}$ in the patients with stage $\mathrm{S} 3$ liver fibrosis $(\mathrm{P}<0.05)$. The threshold of the optimal cut-off value had a sensitivity of $78.6 \%$ and a specificity of $90.4 \%$. These results indicated that the stage of liver fibrosis can be accurately predicted by LSM in patients with CHB with ALT levels <2 times the normal upper limit. In addition, the specificity of prediction is increased with the progression of liver fibrosis.

\section{Discussion}

Liver fibrosis is known to be primarily caused by $\mathrm{CHB}$ viral infection (24). Timely and accurate diagnosis of liver fibrosis is essential to the prevention and treatment for chronic liver disease. Liver biopsy has been considered to be the most reliable method for diagnosing liver fibrosis; however, liver biopsy cannot reflect the conditions of the entire liver, due to the small size of the tissue samples (25). Although numerous patients with $\mathrm{CHB}$ may be persuaded to undergo a first biopsy, the majority will be reluctant to accept follow-up biopsies for the evaluation of disease progression or response to treatment (26). Non-invasive methods of assessing liver fibrosis in a range of chronic liver diseases are currently being investigated (27). Principal among these methods are transient elastography and the use of serum markers, which have already been evaluated in patients with CHB (22). The detection capacity of Fibroscan is $~ 100$-fold greater compared with that of liver biopsy, despite the fact that the accuracy of Fibroscan may be influenced by various factors other than fibrosis, including necroinflammatory activity, obesity, ascites, extrahepatic cholestasis and sampling location (13-15). Previous studies have shown that the area under the ROC curve in patients with CHB with significant fibrosis, advanced fibrosis and cirrhosis were 0.78-0.87, 0.87-0.93 and 0.84-0.96, respectively (28-30). The diagnosis of liver fibrosis and cirrhosis using LSM based on transient elastography has been shown to be superior to the enhanced liver fibrosis test, acoustic radiation force impulse imaging, Forns fibrosis index, Fibrosis-4 index and enhanced liver fibrosis algorithm (22,30-32).

In the present study, LSM was performed with Fibroscan in patients with CHB with ATL levels $<2$ times the normal upper limit. The results indicated that the stage of liver stiffness measured by Fibroscan was positively correlated with the degree of liver fibrosis and necroinflammatory activity measured by liver biopsy. The optimal cut-off values in patients with stage S1, S2 and S3 liver fibrosis were $5.5 \mathrm{kPa}, 8.0 \mathrm{kPa}$, and $10.95 \mathrm{kPa}$, respectively. The area under the ROC curve for the prediction of liver fibrosis development in patients with stage S1, S2 and S3 liver fibrosis was 0.696, 0.911 and 0.923, respectively. In patients with stage S2 and S3 liver fibrosis, Fibroscan was shown to have a higher diagnostic accuracy. The optimal cut-off value exhibited a higher sensitivity and specificity in patients with stage S1, S2 and S3 liver fibrosis, as did the threshold of the optimal cut-off value.

In conclusion, Fibroscan examination is a quantitative analysis technique, which improves the diagnostic sensitivity of liver fibrosis in patients with CHB with ALT levels $<2$ times the normal upper limit.

\section{Acknowledgements}

This study was supported by a project of the Sichuan Provincial Health Department (grant no. 110161).

\section{References}

1. Williams MJ, Clouston AD and Forbes SJ: Links between hepatic fibrosis, ductular reaction, and progenitor cell expansion. Gastroenterology 146: 349-356, 2014.

2. Friedman SL: Liver fibrosis-from bench to bedside. J Hepatol 38 (Suppl 1): S38-S53, 2003. 
3. Lok AS and McMahon AJ: Chronic hepatitis B. Hepatology 45: 507-539, 2007

4. Croagh CM and Lubel JS: Natural history of chronic hepatitis B: Phases in a complex relationship. World J Gastroenterol 20: 10395-10404, 2014.

5. Ginès P, Cárdenas A, Arroyo V and Rodés J: Management of cirrhosis and ascites. N Engl J Med 350: 1646-1654, 2004.

6. Hernandez-Gea V and Friedman SL: Pathogenesis of liver fibrosis. Annu Rev Pathol 6: 425-456, 2011.

7. Rockey DC, Caldwell SH, Goodman ZD, Nelson RC and Smith AD; American Association for the Study of Liver Diseases: Liver biopsy. Hepatology 49: 1017-1044, 2009.

8. Regev A, Berho M, Jeffers LJ Milikowski C, Molina EG, Pyrsopoulos NT, Feng ZZ, Reddy KR and Schiff ER: Sampling error and intraobserver variation in liver biopsy in patients with chronic HCV infection. Am J Gastroenterol 97: 2614-2618, 2002.

9. Bravo AA, Sheth SG and Chopra S: Liver biopsy. N Engl J Med 344: 495-500, 2001.

10. Bedossa P, Dargère D and Paradis V: Sampling variability of liver fibrosis in chronic hepatitis C. Hepatology 38: 1449-1457, 2003.

11. Sharma P, Dhawan S, Bansal R, Tyagi P, Bansal N, Singla V, Kumar A, Matin A and Arora A: The usefulness of transient elastography by FibroScan for the evaluation of liver fibrosis. Indian J Gastroenterol 33: 445-451, 2014.

12. Castera L, Forns X and Alberti A: Non-invasive evaluation of liver fibrosis using transient elastography. J Hepatol 48: 835-847, 2008.

13. Enomoto M, Morikawa H, Tamori A and Kawada N: Noninvasive assessment of liver fibrosis in patients with chronic hepatitis B. World J Gastroenterol 20: 12031-12038, 2014.

14. Sporea I, Gilja OH, Bota S, Sirli R and Popescu A: Liver elastography - an update. Med Ultrason 15: 304-314, 2013.

15. Lee S and Kim do Y: Non-invasive diagnosis of hepatitis B virus-related cirrhosis. World J Gastroenterol 20: 445-459, 2014.

16. Wu T, Li L, Wang H, Luo X and Ning Q: Transient elastography in hepatitis B virus infection. Liver stiffness discrepancy due to sampling location. Saudi Med J 35: 554-560, 2014.

17. Bota S, Sporea I, Peck-Radosavljevic M, Sirli R, Tanaka H, Iijima H, Saito H, Ebinuma H, Lupsor M, Badea R, et al: The influence of aminotransferase levels on liver stiffness assessed by acoustic radiation force impulse elastography: A retrospective multicentre study. Dig Liver Dis 45: 762-768, 2013.

18. Pratt DS and Kaplan MM: Evaluation of abnormal liver-enzyme results in asymptomatic patients. N Engl J Med 342: 1266-1271, 2000.

19. Prati D, Taioli E, Zanella A, Della Torre E, Butelli S, Del Vecchio E, Vianello L, Zanuso F, Mozzi F, Milani S, et al: Updated definitions of healthy ranges for serum alanine aminotransferase levels. Ann Intern Med 137: 1-10, 2002.
20. Zarski JP, Sturm N, Guechot J, Paris A, Zafrani ES, Asselah T, Boisson RC, Bosson JL, Guyader D, Renversez JC, et al: Comparison of nine blood tests and transient elastography for liver fibrosis in chronic hepatitis C: The ANRS HCEP-23 study. J Hepatol 56: 55-62, 2012.

21. Degos F, Perez P, Roche B, Mahmoudi A, Asselineau J, Voitot H and Bedossa P; FIBROSTIC Study Group: Diagnostic accuracy of FibroScan and comparison to liver fibrosis biomarkers in chronic viral hepatitis: A multicenter prospective study (the FIBROSTIC study). J Hepatol 53: 1013-1021, 2010.

22. Trembling PM, Lampertico P, Parkes J, Tanwar S, Viganò M, Facchetti F, Colombo $M$ and Rosenberg WM: Performance of enhanced liver fibrosis test and comparison with transient elastography in the identification of liver fibrosis in patients with chronic hepatitis B infection. J Viral Hepat 21: 430-438, 2014.

23. Reitman S and Frankel S: A colorimetric method for the determination of serum glutamic oxalacetic and glutamic pyruvic transaminases. Am J Clin Pathol 28: 56-63, 1957.

24. Cohen-Naftaly M and Friedman SL: Current status of novel antifibrotic therapies in patients with chronic liver disease. Therap Adv Gastroenterol 4: 391-417, 2011.

25. Lefkowitch JH: Liver biopsy assessment in chronic hepatitis. Arch Med Res 38: 634-643, 2007.

26. Cadranel JF, Rufat P and Degos F: Practices of liver biopsy in France: Results of a prospective nationwide survey. For the Group of Epidemiology of the French Association for the Study of the Liver (AFEF). Hepatology 32: 477-481, 2000.

27. Papastergiou V, Tsochatzis E and Burroughs AK: Non-invasive assessment of liver fibrosis. Ann Gastroenterol 25: 218-231, 2012.

28. Chen YP, Liang XE, Dai L, Zhang Q, Peng J, Zhu YF, Wen WQ, Chan HL and Hou JL: Improving transient elastography performance for detecting hepatitis B cirrhosis. Dig Liver Dis 44: 61-66, 2012.

29. Chan HL, Wong GL, Choi PC, Chan AW, Chim AM, Yiu KK, Chan FK, Sung JJ and Wong VW: Alanine aminotransferase-based algorithms of liver stiffness measurement by transient elastography (Fibroscan) for liver fibrosis in chronic hepatitis B. J Viral Hepat 16: 36-44, 2009.

30. Stebbing J, Farouk L, Panos G, Anderson M, Jiao LR, Mandalia S, Bower M, Gazzard B and Nelson M: A meta-analysis of transient elastography for the detection of hepatic fibrosis. J Clin Gastroenterol 44: 214-219, 2010.

31. Liu Y, Dong CF, Yang G, Liu J, Yao S, Li HY, Yuan J, Li S, Le X, Lin Y, et al: Optimal linear combination of ARFI, transient elastography and APRI for the assessment of fibrosis in chronic hepatitis B. Liver Int 35: 816-825, 2015.

32. Lee MH, Cheong JY, Um SH, Seo YS, Kim DJ, Hwang SG, Yang JM, Han KH and Cho SW: Comparison of surrogate serum markers and transient elastography (Fibroscan) for assessing cirrhosis in patients with chronic viral hepatitis. Dig Dis Sci 55: 3552-3560, 2010. 